\title{
Association of Serum Thyroid Stimulating Hormone and Free Thyroxine with Urinary Albumin Excretion in Euthyroid Sub- jects with Type 2 Diabetes Mellitus
}

Khalid S Aljabri, MD, FRCPC, FACP ${ }^{*}$, Ibrahim M Alnasser, $M D^{1}$, Facharatz ${ }^{2}$, Samia A Bokhari, MD, SBEM ${ }^{1}$, Muneera A Alshareef, MD, SBIM ${ }^{1}$, Patan $M$ Khan, MD, MRCP1, Abdulla M Mallosho, MD ${ }^{1}$,

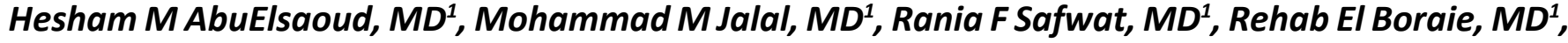
Nawaf K Aljabri, MLT'3 , Bandari K Aljabri, MS ${ }^{4}$, Arwa Y Alsuraihi, MS ${ }^{4}$ and Amjad I Hawsawi, MS ${ }^{4}$

\author{
${ }^{1}$ Department of Endocrinology, King Fahad Armed Forces Hospital, Jeddah, Kingdom of Saudi Arabia \\ ${ }^{2}$ Department of Radiology, King Fahad Armed Forces Hospital, Jeddah, Kingdom of Saudi Arabia \\ ${ }^{3}$ Department of Laboratory, Northern Armed Forces Hospital, Haffr Albatin, Kingdom of Saudi Arabia \\ ${ }^{4}$ College of Medicine, Umm Al Qura University, Makkah, Kingdom of Saudi Arabia
}

*Corresponding author: Khalid S Aljabri, MD, FRCPC, FRCPC (Endo), FACP, ABIM, ABEM, Department of Endocrinology, King Fahad Armed Forces Hospital, Jeddah, PO Box 9862, Jeddah 21159, Kingdom of Saudi Arabia, Tel: +9662323333 ext. 59200

\begin{abstract}
Background and objective: The kidney is responsible for the excretion of thyroid hormones. The present retrospective study was conducted to find out the association of serum thyroid stimulating hormone (TSH) and free thyroxine (FT4) with urinary albumin excretion in euthyroid subjects with type 2 diabetes mellitus (T2DM) in Saudi community based hospital.

Design: We analyzed retrospectively 939 participants whom are between the age 20 to 96 years. All patients were from the population of the Primary health centre at King Fahad Armed Forces Hospital, Jeddah, Saudi Arabia. All data were collected on the basis of a review of electronic medical data. Participants were defined as having T2DM according to self-report, clinical reports, use of antidiabetic agents and $\mathrm{HbA1c}(\geq 6.5)$. HbA1c was expressed as percentage. High performance liquid chromatography was used. Serum FT4 were estimated by radioimmunoassay and Serum TSH was estimated by Immunoradiometric assay. All patients in the present study fulfilled the revised National Kidney Foundation criteria for the diagnosis of albuminuria. The urine albumin to creatinine ratio (ACR) was used as the index of urinary albumin excretion. A urine sample was collected during the first morning voiding. Conventionally, subjects with $A C R<30 \mathrm{mg} / \mathrm{g}$ were defined as having normal
\end{abstract}

albuminuria (NA). ACR between $30-299 \mathrm{mg} / \mathrm{g}$ is called moderately increased albuminuria. Levels $\geq 300 \mathrm{mg} / \mathrm{g}$ are called severely increased albuminuria.

Results: 939 subjects with T2DM were included. There were $299(31.8 \%)$ male and $640(68.2 \%)$ were female with mean age $55.9 \pm 12.5$. The mean TSH and FT4 value were $1.9 \pm 0.9 \mathrm{mIU} / \mathrm{l}$ and $15.8 \pm 2.5 \mathrm{pmol} / \mathrm{l}$ respectively. Among cases of T2DM and albuminuria, there were $608(64.7 \%)$ with NA, $262(27.9 \%)$ with moderately increased albuminuria and $70(7.4 \%)$ with severly increased albuminuria. Patients with severely increased albuminuria were significantly older than patients with NA and moderately increased albuminuria, $58.9 \pm 9.9$ vs. $55.1 \pm 12.4$ and $56.9 \pm 13.1$ respectively, $p=0.02$. Frequency of female patients were higher across all albuminuria grades, $p=0.001$. Patients with severely increased albuminuria were significantly have higher TSH than patients with NA and moderately increased albuminuria, $2.1 \pm 0.9$ vs. $1.8 \pm 0.9$ and $1.9 \pm 0.9$ respectively, $p=0.02$. Patients with severely increased albuminuria were significantly have lower FT4 than patients with NA and moderately increased albuminuria, $15.3 \pm 1.9$ vs. $16.1 \pm 2.5$ and $15.4 \pm 2.6$ respectively, $p=0.045$. Patients with severely increased albuminuria have higher TSH than patients with NA and moderately increased albuminuria across all age groups. Patients with severely increased albuminuria have lower FT4 than patients with NA and moderately increased albuminuria across all age groups.

Citation: Aljabri KS, Alnasser IM, Bokhari SA, Alshareef MA, Khan PM, et al. (2019) Association of Serum Thyroid Stimulating Hormone and Free Thyroxine with Urinary Albumin Excretion in Euthyroid Subjects with Type 2 Diabetes Mellitus. Int J Diabetes Clin Res 6:102. doi.org/10.23937/2377-3634/1410102 Accepted: March 18, 2019: Published: March 20, 2019

Copyright: (c) 2019 Aljabri KS, et al. This is an open-access article distributed under the terms of the Creative Commons Attribution License, which permits unrestricted use, distribution, and reproduction in any medium, provided the original author and source are credited. 
Conclusion: We conclude that despite the limitations of this hospital-based retrospective study, high TSH and low FT4 levels are highly prevalent in cohort of Saudis with albuminuria and T2DM. The majority of our patients in our finding were predominantly females. These two observations remain to be validated by population-based studies. In the absence of registry data, larger cooperative studies involving diverse population samples from multiple centers could help to provide further information on the true thyroid hormone abnormalities.

\section{Keywords}

Albuminuria, Thyroid hormones, Type 2 diabetes, Saudi Arabia

\section{Introduction}

Diabetes Mellitus is the commonest endocrine disorder [1]. Saudi Arabia is the seventh of the top ten countries in terms of the prevalence of type 2 diabetes mellitus (T2DM) among Saudi adults older than 20 years [2]. According to the 2010 Global Burden of Disease study, chronic kidney disease (CKD) was ranked $27^{\text {th }}$ in the list of causes of total number of deaths worldwide in 1990 , but rose to 18th in 2010 [3]. The natural history of diabetic nephropathy (DN) from prospective data is less well described for T2DM [4]. The earliest clinical sign of $\mathrm{DN}$, defined as microalbuminuria which is an elevated urinary excretion of albumin $[5,6]$.

Thyroid hormones play a very important role regulating metabolism, development, protein synthesis, and influencing other hormone functions. The two main hormones produced by the thyroid are triiodothyronine (T3) and thyroxine (T4). These hormones can also have significant impact on kidney disease so it is important to consider the physiological association of thyroid dysfunction in relation to CKD. Thyroid stimulating hormone (TSH) which is biosynthesized in pituitary is responsible for every single biochemical process within thyroid gland including T4 and T3 production [7]. It seems eventually kidney is involved in key pathways of the T4 and T3 metabolism and any condition with eventual renal dysfunction may lead to thyroid disorder and this abnormality can be manifested through thyroid hormones production and metabolism. Thyroid hormone excretion by the kidney can be considered as in how the kidney affect on thyroid hormones with eventual interfering with thyroid gland metabolism and it is believed that kidney deviation from its normal function, eventually adversely affect thyroid gland and lead to thyroid malfunction $[8,9]$.

It is well documented that the kidney also can have influences on the way thyroid function. The kidney is responsible for metabolism of thyroid hormones through the degradation and excretion of thyroid hormones by renal function. Therefore in case of kidney diseases, thyroid disorder may be encountered negatively but this process is not straight forward strategy and thyroid abnormalities mainly occur, when
CKD are present [10,11]. Gilles, et al. made the interesting observation that patients with proteinuria had higher TSH levels, which can be explained by the possible loss of thyroid hormones in the urine [12]. In addition, thyroid hormone abnormalities have been reported among euthyroid patients with ESRD, including reduced total and free triiodothyronine and thyroxine levels [13]. Reasons for these latter findings are unclear, but it has been postulated to be due, at least in part, to an adaptive response to chronic nonthyroidal illness, unresolved uremia, and protein malnutrition [14].

Thus, the present retrospective study was conducted to find out the thyroid hormone abnormalities in patients with albuminuria and type 2 diabetes mellitus in Saudi community based hospital.

\section{Methods}

We analyzed retrospectively 939 participants whom are between the age 20 to 96 years. All patients were from the population of the Primary health centre at King Fahad Armed Forces Hospital, Jeddah, Saudi Arabia. All data were collected on the basis of a review of electronic medical data. Participants were defined as having T2DM according to self-report, clinical reports, use of antidiabetic agents and $\mathrm{HbA} 1 \mathrm{c}(\geq 6.5)$ [5]. HbA1c was expressed as percentage. High performance liquid chromatography was used for $\mathrm{HbA} 1 \mathrm{c}$ measurement. Serum FT4 were estimated by radioimmunoassay and Serum TSH was estimated by Immunoradiometric assay. All patients in the present study fulfilled the revised National Kidney Foundation criteria for the diagnosis of albuminuria [6]. The urine albumin to creatinine ratio $(A C R)$ was used as the index of urinary albumin excretion. A urine sample was collected during the first morning voiding. Conventionally, subjects with ACR < $30 \mathrm{mg} / \mathrm{g}$ were defined as having normal albuminuria (NA). ACR between $30-299 \mathrm{mg} / \mathrm{g}$ is called moderately increased albuminuria. Levels $\geq 300 \mathrm{mg} / \mathrm{g}$ are called severely increased albuminuria [15-17]. A polyclonal radioimmunoassay was used for albumin measurement. The total number of cohort were separated on basis of age values into four groups: $<40$ years, $40-49$ years, 50 59 years and $\geq 60$ years.

\section{Statistical Analysis}

Continuous variables were described using means and standard deviations. Univariate analysis of baseline demography both between groups, were accomplished using unpaired t-test and Chi square test were used for categorical data comparison. Pearson correlation was used for correlation. P value $<0.05$ indicates significance. The statistical analysis was conducted with SPSS version 22.0 for Windows.

\section{Results}

939 subjects with T2DM were included. There were $299(31.8 \%)$ male and 640 (68.2\%) were female with 
mean age $55.9 \pm 12.5$, Table 1 . The mean TSH and FT4 value were $1.9 \pm 0.9 \mathrm{mIU} / \mathrm{l}$ and $15.8 \pm 2.5 \mathrm{pmol} / \mathrm{l}$ respectively. Among cases of T2DM and albuminuria, there were $608(64.7 \%)$ with NA, 262 (27.9\%) with

Table 1: Base line characteristic of patients with type 2 diabetes [mean \pm standard deviation or number (\%)].

\begin{tabular}{|c|c|c|}
\hline \multicolumn{2}{|c|}{ Parameters } & Total (939) \\
\hline \multicolumn{2}{|c|}{ Age (years) } & $55.9 \pm 12.5$ \\
\hline \multirow[t]{2}{*}{ Gender } & Male & $299(31.8)$ \\
\hline & Female & $640(68.2)$ \\
\hline \multicolumn{2}{|c|}{ Albumin creatinine ratio $(\mathrm{mg} / \mathrm{g})$} & $98.7 \pm 305.4$ \\
\hline \multicolumn{2}{|c|}{ HbA1c (\%) } & $8.2 \pm 2.2$ \\
\hline \multicolumn{2}{|c|}{ TSH (mIU/l) } & $1.9 \pm 0.9$ \\
\hline \multicolumn{2}{|c|}{ FT4 (pmol/l) } & $15.8 \pm 2.5$ \\
\hline \multicolumn{2}{|c|}{ Serum creatinine $(\mu \mathrm{mol} / \mathrm{L})$} & $70.2 \pm 25.2$ \\
\hline
\end{tabular}

moderately increased albuminuria and 70 (7.4\%) with severly increased albuminuria, Table 2. Patients with severely increased albuminuria were significantly older than patients with NA and moderately increased albuminuria, $58.9 \pm 9.9$ vs. $55.1 \pm 12.4$ and $56.9 \pm 13.1$ respectively, $p=0.02$. Frequency of female patients were higher across all albuminuria grades, $p=0.001$. Patients with severely increased albuminuria were significantly have higher TSH than patients with NA and moderately increased albuminuria, $2.1 \pm 0.9$ vs. $1.8 \pm 0.9$ and 1.9 \pm 0.9 respectively, $p=0.02$. Patients with severely increased albuminuria were significantly have lower FT4 than patients with NA and moderately increased albuminuria, $15.3 \pm 1.9$ vs. $16.1 \pm 2.5$ and $15.4 \pm 2.6$ respectively, $p=0.045$. Patients with severely increased albuminuria have higher TSH than patients with NA and moderately increased albuminuria across all age groups, Figure 1. Patients with severely increased albuminuria

Table 2: Comparison between patients with type 2 diabetes with and without albuminuria [mean \pm standard deviation or number $(\%)]$.

\begin{tabular}{|c|c|c|c|c|c|}
\hline \multicolumn{2}{|c|}{ Parameters } & \multicolumn{3}{|c|}{ Albuminuria (mg/g) } & \multirow[t]{3}{*}{$P$ value } \\
\hline & & \multirow{2}{*}{\begin{tabular}{|l|}
$<30$ \\
$608(64.7)$ \\
\end{tabular}} & \multirow{2}{*}{\begin{tabular}{|l|}
$30-299$ \\
$262(27.9)$
\end{tabular}} & \multirow{2}{*}{$\begin{array}{l}\geq 300 \\
70(7.4)\end{array}$} & \\
\hline & & & & & \\
\hline \multicolumn{2}{|c|}{ Age (years) } & $55.1 \pm 12.4$ & $56.9 \pm 13.1$ & $58.9 \pm 9.9$ & 0.02 \\
\hline \multirow[t]{2}{*}{ Gender } & Male & $169(27.8)$ & $102(38.9)$ & $28(40.6)$ & \multirow[t]{2}{*}{0.001} \\
\hline & Female & $439(72.2)$ & $160(61.1)$ & $41(59.4)$ & \\
\hline \multicolumn{2}{|c|}{ Albumin creatinine ratio $(\mathrm{mg} / \mathrm{g})$} & $10.1 \pm 7.2$ & $83.2 \pm 56.1$ & $936.7 \pm 699.2$ & $<0.0001$ \\
\hline \multicolumn{2}{|c|}{$\mathrm{HbA1c}(\%)$} & $7.9 \pm 2.1$ & $8.6 \pm 2.2$ & $9.2 \pm 2.1$ & $<0.0001$ \\
\hline \multicolumn{2}{|c|}{ TSH (mIU/l) } & $1.8 \pm 0.9$ & $1.9 \pm 0.9$ & $2.1 \pm 0.9$ & 0.03 \\
\hline \multicolumn{2}{|c|}{ FT4 (pmol/l) } & $16.1 \pm 2.5$ & $15.4 \pm 2.6$ & $15.3 \pm 1.9$ & 0.045 \\
\hline \multicolumn{2}{|c|}{ Serum creatinine $(\mu \mathrm{mol} / \mathrm{L})$} & $66.5 \pm 21.8$ & $72.6 \pm 24.2$ & $93.2 \pm 40.0$ & $<0.0001$ \\
\hline
\end{tabular}

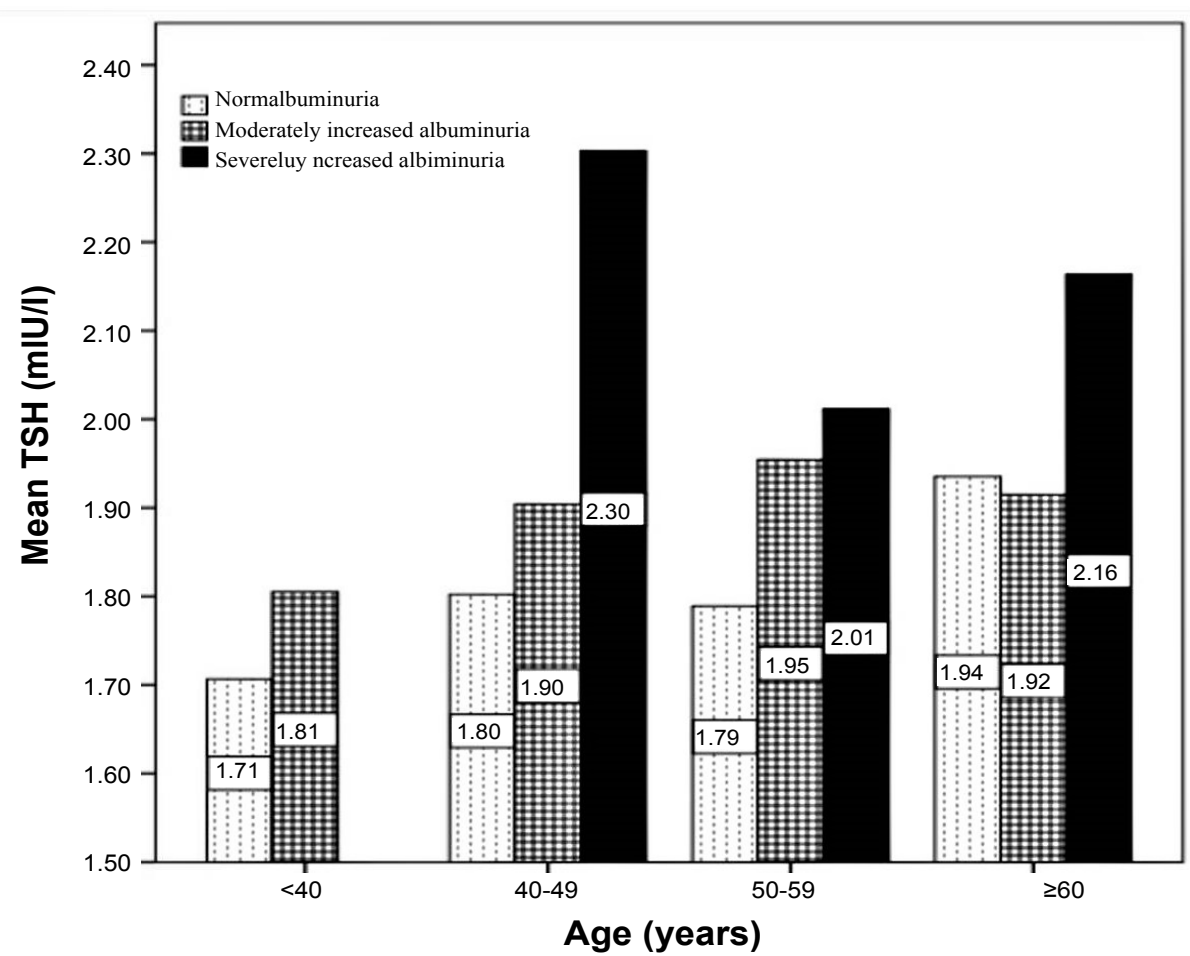

Figure 1: Age category groups and the mean TSH in patients with type 2 diabetes with and without albuminuria. 
have lower FT4 than patients with NA and moderately increased albuminuria across all age groups, Figure 2.

\section{Discussion}

Our study demonstrated that abnormalities in thyroid function occur in patients with T2DM and proteinuria. Specifically, TSH levels were higher and FT4 levels were lower in patients with albuminuric diabetic renal diseases. The interplays between thyroid and kidney have been recognized in many disease states. Thyroid dysfunction can influence kidney through the immunemediated pathway and thyroid hormones [18]. The thyroid disorder and coincident nephropathy have been reported mainly in the patients presented with albuminuria $[19,20]$. In this situation, thyroid hormone may influence glomerular and tubular functions through pre-renal and intrinsic renal effects. Thyroid hormones influence renal development, kidney structure, renal hemodynamics, glomerular filtration rate, the function of many transport systems along the nephron and sodium and water homeostasis. These effects of thyroid hormone are due to direct renal actions and in part are mediated by cardiovascular and systemic hemodynamic effects that influence kidney function. Disorders of thyroid function have also been linked to development of immune mediated glomerular injury and alterations in thyroid hormones and thyroid hormone testing occur in patients with kidney disease.

Higher TSH levels are seen with increasing age [21]. The mean age for patients with severely increased albuminuria, NA and moderately increased albuminuria in this study was $58.9 \pm 9.9,55.1 \pm 12.4$ and $56.9 \pm 13.1$ years respectively, $p=0.02$.

The TSH level is often elevated in CKD in response to TSH from pituitary as a result of uremic effect [22]. In agreement to our study, patients with severely increased albuminuria has the worst HbA1c compared to NA and moderately increased albuminuria patients, $9.2 \pm 2.1$ vs. $8.6 \pm 2.2$ and $7.9 \pm 2.1$ respectively, $p<$ 0.0001 [23]. TSH also loses its circadian rhythm along with compromised bioactivity due to poor glycosylation. The Wolffe Chaikoff effect has been cited as a causative phenomenon behind the rise of this disorder in diabetic kidney disease patients [24].

In agreement to our study, we found that patients with severely increased albuminuria were significantly have lower FT4 than patients with NA and moderately increased albuminuria, $15.3 \pm 1.9$ vs. $16.1 \pm 2.5$ and

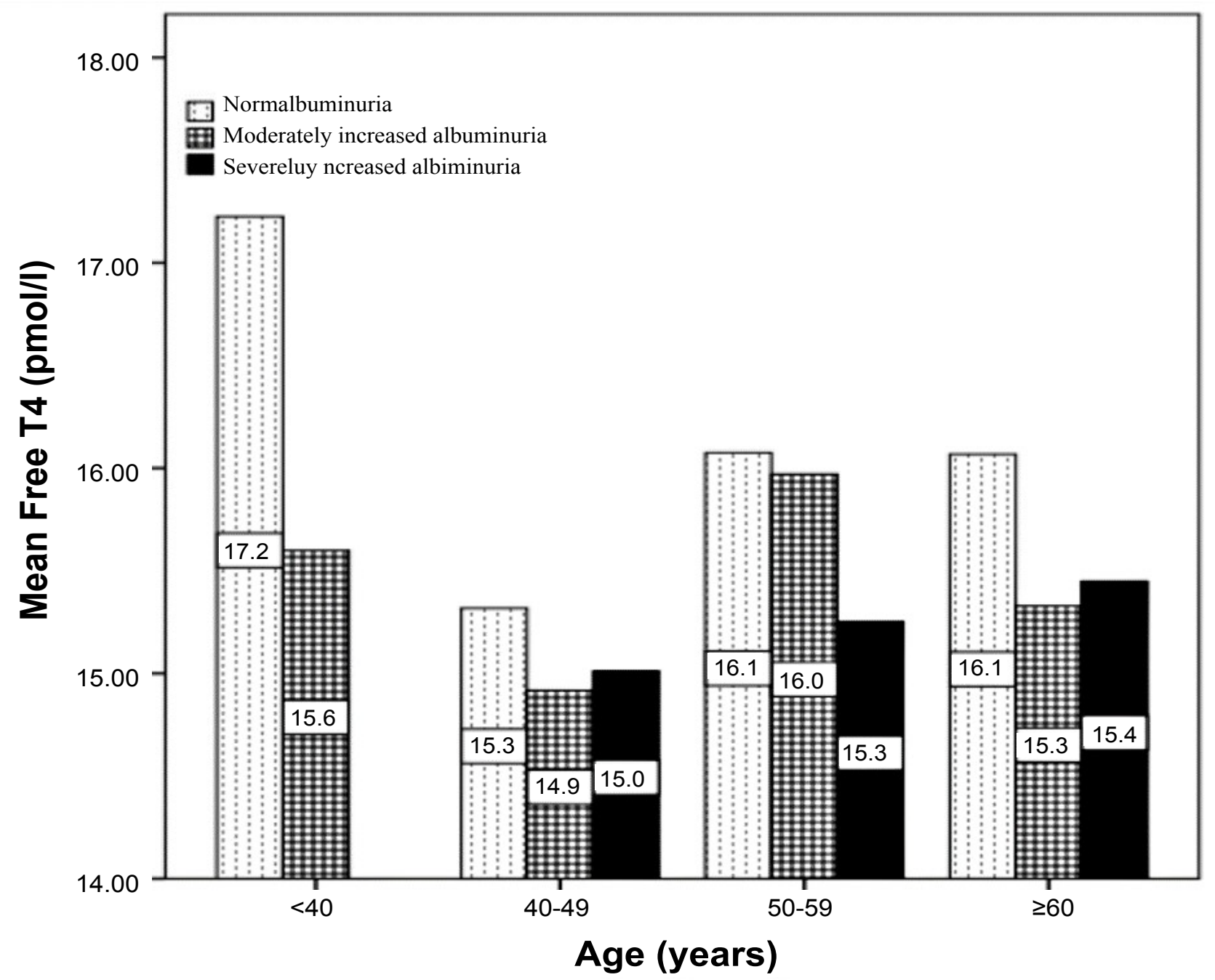

Figure 2: Age category groups and the mean Free T4 in patients with type 2 diabetes with and without albuminuria. 
$15.4 \pm 2.6$ respectively, $p=0.045$ [12]. The role of albuminuria is confirmed by the significant positive correlation between TSH and albuminuria, $r=0.08, p$ $=0.01$ and nonsignificant negative correlation between FT4 and albuminuria, $r=-0.07, p=0.2$. Proteinuria is a hallmark of renal diseases. Severe albuminuria results in hypoalbuminaemia. Albumin is the most abundant protein in serum and urine. In patients with albuminuria many other proteins beside albumin are lost in the urine. Among these are hormones and hormonebinding proteins. Several studies have documented urinary loss of thyroid hormones and thyroxin-binding globulin (TBG) in patients with albuminuria [25-28]. In patients with the nephrotic syndrome, loss of thyroid hormones may lead to low free thyroid hormone levels unless production is increased under the influence of TSH. Furthermore, loss of albumin and TBG may reduce the binding capacity for thyroid hormones, resulting in a decrease in total T4 concentrations.

We aimed to identify the levels of thyroid hormones in patients with euthyroid T2DM complicated with albuminuria in primary health care setting. Furthermore, due to the retrospective nature of this study, the observed population reflects a selected yet comprehensive group of patients rather than the general population. Our study could be limited by the question of clustering of cases within the study region and the effect that might have on our estimates, in addition, the current study population may appear limited in size and therefore may underestimate the true thyroid hormone abnormailities in patients with T2DM complicated with albuminuria. In addition, the study shares the limitations of all retrospective studies.

We conclude that despite the limitations of this hospital-based retrospective study, high TSH and low FT4 levels are highly prevalent in cohort of Saudis with albuminuria and T2DM. The majority of our patients in our finding were predominantly females. These two observations remain to be validated by populationbased studies. In the absence of registry data, larger cooperative studies involving diverse population samples from multiple centers could help to provide further information on the true thyroid hormone abnormalities.

\section{Acknowledgement}

The author would like to thank all colleagues from the Department of primary care for helping in data collection.

\section{Conflict of Interests}

The authors declare no conflict of interests.

\section{References}

1. Faghilimnai S, Hashemipour M, Kelishadi B (2006) Lipid profile of children with type 1 diabetes compared to controls. ARYA J 2: 36-38.

2. IDF (2012) Diabetes Atlas ( $5^{\text {th }}$ edn).
3. Jha V, Garcia G, Iseki K, Li Z, Naicker S, et al. (2013) Chronic kidney disease: Global dimension and perspectives. Lancet 382: $260-272$.

4. Parving $\mathrm{HH}$ (1996) Initiation and progression of diabetic nephropathy. N Engl J Med 335: 1682-1683.

5. American Diabetes Association (2019) Classification and diagnosis of diabetes: Standards of medical care in diabetes-2019. Diabetes Care 42: S13-S28.

6. Mogensen CE, Chachati A, Christensen CK, Close CF Deckert T, et al. (1985) Microalbuminuria: An early marker of renal involvement in diabetes. Uremia Invest 9: 85-95.

7. Adriaanse R, Brabant G, Endert E, Wiersinga WM (1993) Pulsatile TSH release in patients with untreated pituitary disease. J Clin Endocrinol Metab 77: 205-209.

8. Kaptein EM, Hoopes MT, Parise M, Massry SG (1991) rT3 metabolism in patients with nephrotic syndrome and normal GFR compared with normal subjects. Am J Physiol 260: E641-E650.

9. Katz Al, Emmanouel DS, Lindheimer MD (1975) Thyroid hormone and the kidney. Nephron 15: 223-249.

10. Chonchol M, Lippi G, Salvagno G, Zoppini G, Muggeo $M$, et al. (2008) Prevalence of subclinical hypothyroidism in patients with chronic kidney disease. Clin J Am Soc Nephrol 3: 1296-1300.

11. Lo JC, Chertow GM, Go AS, Hsu CY (2005) Increased prevalence of subclinical and clinical hypothyroidism in persons with chronic kidney disease. Kidney Int 67: 10471052.

12. Gilles R, den Heijer M, Ross AH, Sweep FC, Hermus AR, et al. (2008) Thyroid function in patients with proteinuria. Neth J Med 66: 483-485.

13. Hegedus L, Andersen JR, Poulsen LR, Perrild H, Holm B, et al. (1985) Thyroid gland volume and serum concentrations of thyroid hormones in chronic renal failure. Nephron 40: 171-174.

14. Lim VS (2001) Thyroid function in patients with chronic renal failure. Am J Kidney Dis 38: S80-S84.

15. National Kidney Foundation (2002) K/DOQI clinical practice guidelines for chronic kidney disease. Part 5: Evaluation of laboratory measurements for clinical assessment of kidney disease. Am J Kidney Dis 39: S76-S92.

16. Isakova T, Nickolas TL, Denburg M, Yarlagadda S, Weiner DE, et al. (2017) KDOQI US commentary on the 2017 KDIGO clinical practice guideline update for the diagnosis, evaluation, prevention, and treatment of Chronic Kidney Disease-Mineral and Bone Disorder (CKD-MBD). Am J Kidney Dis 70: 737-751.

17. Eknoyan G, Hostetter T, Bakris GL, Hebert L, Levey AS, et al. (2003) Proteinuria and other markers of chronic kidney disease: A position statement of the national kidney foundation (NKF) and the national institute of diabetes and digestive and kidney diseases (NIDDK). Am J Kidney Dis 42: 617-622.

18. Basu G, Mohapatra A (2012) Interactions between thyroid disorders and kidney disease. Indian J Endocrinol Metab 16: 204-213.

19. Kocak G, Huddam B, Azak A, Ortabozkoyun L, Duranay M (2012) Coexistent findings of renal glomerular disease with Hashimoto's thyroiditis. Clin Endocrinol (Oxf) 76: 759-762.

20. Hao Y, Wu X, Huang X, Tang Z (2017) Proteinuria associated with thyroid disorders-cases report and literature review. Med Case Rep 3: 35. 
21. Surks MI, Hollowell JG (2007) Age-specific distribution of serum thyrotropin and antithyroid antibodies in the US population: Implications for the prevalence of subclinical hypothyroidism. J Clin Endocrinol Metab 92: 4575-4582.

22. Ramirez G, O'Neill Jr W, Jubiz W, Bloomer HA (1976) Thyroid dysfunction in uremia: Evidence for thyroid and hypophyseal abnormalities. Ann Intern Med 84: 672-676.

23. Chandra A (2016) Prevalence of hypothyroidism in patients with chronic kidney disease: A cross-sectional study from North India. Kidney Res Clin Pract 35: 165-168.

24. Bando Y, Ushiogi Y, Okafuji K, Toya D, Tanaka N, et al. (2002) Non-autoimmune primary hypothyroidism in diabetic and non-diabetic chronic renal dysfunction. Exp Clin Endocrinol Diabetes 110: 408-415.

25. Adlkofer F, Hain H, Meinhold H, Kraft D, Ramsden D, et al. (1983) Thyroid function in patients with proteinuria and normal or increased serum creatinine concentration. Acta Endocrinol (Copenh) 102: 367-376.

26. Afrasiabi MA, Vaziri ND, Gwinup G, Mays DM, Barton $\mathrm{CH}$, et al. (1979) Thyroid function studies in the nephrotic syndrome. Ann Intern Med 90: 335-338.

27. Fonseca V, Thomas $M$, Katrak A, Sweny $P$, Moorhead JF (1991) Can urinary thyroid hormone loss cause hypothyroidism? Lancet 338: 475-476.

28. Liappis N, Rao GS (1985) Behavior of the levels of free triiodothyronine, triiodothyronine, free thyroxine, thyroxine, thyrotropin and thyroxinebinding globulin in the serum of children with nephrotic syndrome. Klin Padiatr 197: 423426. 